\title{
Gallstone Ileus: An Unfortunate Complication after Endoscopic Biliary Drainage
}

\author{
Dinesh Baithma Jothi ${ }^{1,2}$, Dexter Lai Zen Ran ${ }^{2,3}$, Sanjeev Sandrasecra ${ }^{4}$, Firdaus Hayati ${ }^{5 *}$ \\ ${ }^{1}$ Department of Surgery, Faculty of Medicine, University of Malaya, Kuala Lumpur, Malaysia; ${ }^{2}$ Department of Surgery, Queen \\ Elizabeth Hospital, Ministry of Health Malaysia, Kota Kinabalu, Sabah, Malaysia; ${ }^{3}$ Department of Surgery, Faculty of Medicine, \\ Universiti Kebangsaan Malaysia Medical Centre, Kuala Lumpur, Malaysia; ${ }^{4}$ Department of Surgery, Selayang Hospital, Ministry \\ of Health Malaysia, Selayang, Selangor, Malaysia; ${ }^{5}$ Department of Surgery, Faculty of Medicine and Health Sciences, Universiti \\ Malaysia Sabah, Kota Kinabalu, Sabah, Malaysia
}

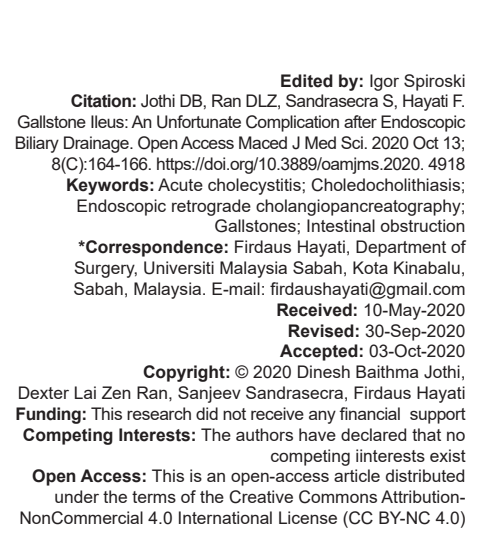

\section{Abstract}

BACKGROUND: Gallstone ileus is a misnomer of intestinal obstruction. This rare entity of gallstone disease happens after episodes of acute cholecystitis, Mirizzi's syndrome, or even after endoscopic intervention.

CASE REPORT: A 17-year-old lady with acute cholecystitis, hepatolithiasis, and choledocholithiasis was endoscopically managed through endoscopic retrograde cholangiopancreatography. She was initially well but complicated with intestinal obstruction the next day. The abdominal radiograph and computed tomography showed a dilated small bowel secondary to gallstone ileus. An emergency laparotomy was performed to relieve the obstruction.

CONCLUSION: Gallstone ileus is a rare cause of intestinal obstruction. It must be suspected in a patient with underlying gallstone disease, especially after an endoscopic biliary intervention. Emergency enterotomy and stone extraction are the imperative surgical intervention if the stone is not able to pass out spontaneously.

\section{Background}

Gallstone ileus is a known yet rare complication of cholelithiasis. This entity occurs in $<0.5 \%$ of the patients with gallstone diseases [1]. It is, in fact, a misnomer as the terminology refers to a mechanical obstruction instead of a functional obstruction. In the literature, it is about $1-4 \%$ of all known cases of intestinal obstruction [2]. The youngest reported case involved a 13-year-old patient, while the oldest case is a 99-year-old patient [3], [4]. According to the predominant association of female gender in gallstone diseases, the majority of gallstone ileus patients correspond well to the female population. We discuss a 17 -year-old lady who was investigated for obstructive jaundice, complicated with intestinal obstruction after endoscopic retrograde cholangiopancreatography (ERCP), and we study its literature review.

\section{Case Report}

A 17-year-old girl without a previous medical history presented with epigastric pain for 2 weeks duration.
She denied fever, nausea, vomiting, chills, or rigors. There was no history of ingesting traditional medicine, family history of thalassemia, glucose-6-phosphate dehydrogenase deficiency, or hemolytic anemia. On examination, she was comfortable but jaundiced. There were no stigmata of chronic liver disease, abdominal tenderness, and palpable mass. Ultrasonography (USG) of the abdomen showed the features of acute cholecystitis with hepatolithiasis, choledocholithiasis, and mild biliary obstruction. Computed tomography (CT) of the liver confirmed similar findings. An ERCP was performed for the patient using a routine technique. It showed a dilated common bile duct (CBD) with dislodged stone in the second part of the duodenum. The CBD was cannulated with a biliary stent. The procedure went well without any immediate perioperative complication.

However, a day after the ERCP, the patient started to complain of abdominal pain, associated with persistent vomiting and no bowel opening. The abdominal radiograph revealed a dilated small bowel. Thus, CT of the abdomen was arranged, which showed features of gallstone ileus. Two large calculi were located in the distal ileum causing proximal small bowel dilatation Figure 1. An emergency laparotomy was performed to relieve the obstruction. Intraoperatively, we noted 2 large stones 


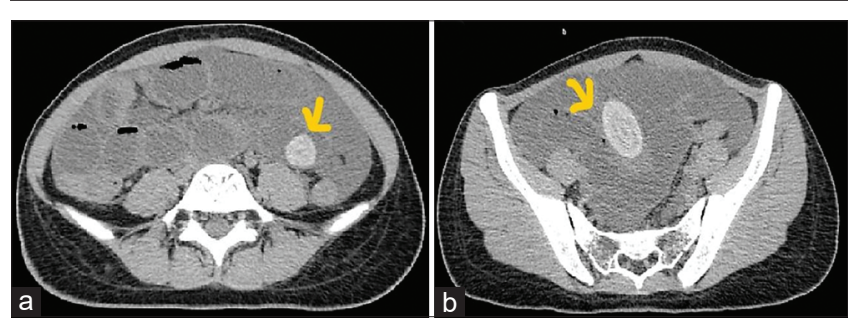

Figure 1: (a) Hyperdense rounded lesion (arrow) noted in the small bowel with proximal bowel dilatation. (b) Larger sized hyperdense oval lesion (arrow) in the pelvic cavity

impacted at the distal ileum measuring $4 \mathrm{~cm} \times 3 \mathrm{~cm}$ and $2 \mathrm{~cm} \times 2 \mathrm{~cm}$, respectively, whereby an enterotomy, bowel decompression, and stone removal were performed Figure 2. The patient was recovering well postoperatively with no complications. She was discharged home on the day 4 post-operation. She was planned for cholecystectomy and $\mathrm{CBD}$ reconstruction on a later date.

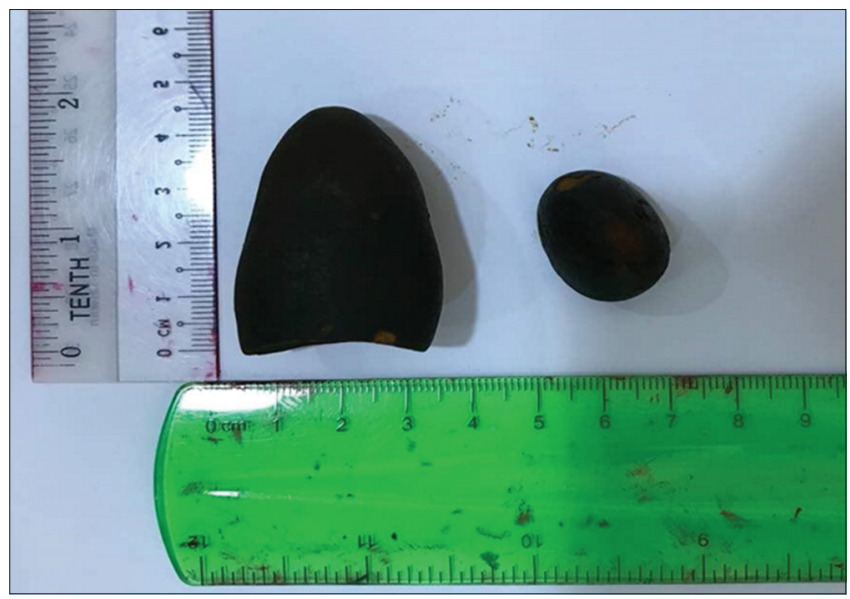

Figure 2: Two large stones retrieved during laparotomy which impacted at the distal ileum

\section{Discussion}

Gallstone ileus is postulated to be one of the rarest complications of gallstone diseases. It frequently occurs after episodes of acute cholecystitis and Mirizzi's syndrome. The concurrent inflammation and intrabiliary pressure cause erosion through the gallbladder wall, leading to a formation of a fistula between the wall of the gallbladder and the adjacent gastrointestinal visceral organs [1]. Subsequently, the continuous and persistent pressure effect will lead to the passage of the gallstone through the biliary-enteric fistula. In addition, the gallstone may also pass to the duodenum through a dilated papilla of Vater caused by a sphincterotomy. Once within the gastrointestinal tract, the gallstone usually proceeds distally and may pass out spontaneously through the rectum, or it may become impacted and cause obstruction. The latter happened to our patient after ERCP.

The obstruction can occur almost in any part of the gastrointestinal tract. Most common sites of the obstruction are at the terminal ileum and ileocecal valve due to their smaller luminal caliber and less active peristalsis [1]. Less frequently, if the gallstones are large enough, they may be impacted in the proximal bowel tract, such as the ileum, jejunum, and even stomach and duodenum (Bouveret's syndrome). Based on the previous literature, the impacted gallstones ranged from 2 to $10 \mathrm{~cm}$ in size with a mean of $4.3 \mathrm{~cm}$ [1]. Gallstones larger than $5 \mathrm{~cm}$ are more likely to become impacted, although the spontaneous passage of such big gallstones has been reported. The largest gallstone ileus ever extracted measures $17.7 \mathrm{~cm}$ in its largest diameter and it was removed from the transverse colon [2]. In our case, the gallstones are similar to the other reported cases as the stones are within the normal mean size, and they were extracted from the terminal ileum.

Patients with gallstone ileus usually would present initially with manifestations of biliary obstruction, including right hypochondrial pain, fever with chill and rigor, or jaundice [1]. The symptoms are suggestive of the existence of gallstone before it dislodges or fistulates into the gastrointestinal tract. The moment it causes intestinal obstruction, the classical triad of colicky abdominal pain with distension, vomiting, and absence of bowel output will emerge. The physical findings are mostly non-specific. The patients often have dehydration, distended, and tender abdomen with high-pitched bowel sound. Occasionally, they can appear icteric as seen in our patient. Clinical toxicity, hemodynamic instability, and peritonitis may be noted if perforation follows.

Imaging plays a huge role in establishing the correct diagnosis. Rigler and Balthazar had described five radiographic signs on plain abdominal radiography in gallstone ileus, namely, (1) partial or complete intestinal obstruction, (2) pneumobilia or contrast material in the biliary tree, (3) an aberrant gallstone, (4) change of the position of such gallstone on serial films, and (5) air-fluid levels in the right upper quadrant corresponds to the duodenum and lateral to the gallbladder [5], [6]. USG will be an adjunct imaging modality to visualize complicated gallstone diseases such as cholecystitis, fistula, impacted stone, and choledocholithiasis. The use of USG in combination with abdominal radiograph can increase the sensitivity of the diagnostic modality by up to $74 \%$ [7] Another important imaging is CT scan which provides a superior result than the rest. It is paramount to locate the fistula, offending gallstones and intestinal obstruction with imaging precision, and aid in therapeutic decision making.

The management of gallstone disease involves a multiphase approach. The etiology of jaundice must be tackled first. In our case, an ERCP was performed to relieve the biliary obstruction. However, it was complicated with stone dislodgement causing intestinal obstruction. Fluid resuscitation is of the utmost importance as dehydration can be devastating to the patient. The treatment of gallstone ileus mostly involves the removal of stone to relieve the acute intestinal obstruction. Emergency laparotomy is necessary if the stone is not able to pass out spontaneously. 
Enterotomy and stone extraction alone remains the standard operation. Gallstone ileus is benign, but it carries a poor prognosis if neglected, especially among old age. The previous studies reported that the mortality rate is as high as $15 \%$ largely due to delayed diagnosis and comorbid conditions such as cardiorespiratory disease, obesity, and diabetes mellitus [1]. The subsequent operation is needed for the biliary pathology, which is a cholecystectomy to resect the stone reservoir.

\section{Conclusion}

Gallstone ileus is a rare cause of intestinal obstruction. It must be considered as a differential diagnosis in a patient with underlying gallstone disease, especially after an endoscopic biliary intervention. CT of the abdomen is superior to the plain abdominal radiography and USG in therapeutic decision making. Emergency enterotomy and stone extraction are the imperative surgical intervention if the stone is not able to pass out spontaneously.

\section{Acknowledgments}

We would like to thank the Director General of Health Malaysia for his permission to publish this article as a case report.

\section{References}

1. Nuño-Guzmán CM, Marín-Contreras ME, Figueroa-Sánchez M, Corona JL. Gallstone ileus, clinical presentation, diagnostic and treatment approach. World J Gastrointest Surg. 2016;8(1):6576. https://doi.org/10.4240/wjgs.v8.i1.65 PMid:26843914

2. Abich E, Glotzer D, Murphy E. Gallstone ileus: An unlikely cause of mechanical small bowel obstruction. Case Rep Gastroenterol. 2017;11(2):389-95. https://doi.org/10.1159/000475749 PMid:29033757

3. Nakao A, Okamoto Y, Sunami M, Fujita T, Tsuji T. The oldest patient with gallstone ileus: Report of a case and review of 176 cases in Japan. Kurume Med J. 2008;55(1-2):29-33. https://doi. org/10.2739/kurumemedj.55.29

PMid:18981682

4. Sánchez AM, Stringel GB, Martínez AT, Fernández CS Camacho LD, Sánchez RM. Surgical management of gallstone ileus: Fourteen year experience. Rev Gastroenterol Mex. 2005;70(1):44-9.

PMid:16170962

5. Rigler LG, Borman CN, Noble JF. Gallstone obstruction: Pathogenesis and roentgen manifestations. JAMA. 1941;117(21):1753-9. https://doi.org/10.1001/ jama.1941.02820470001001

6. Balthazar EJ, Schechter LS. Air in gallbladder: A frequent finding in gallstone ileus. AJR Am J Roentgenol. 1978;131(2):219-22. https://doi.org/10.2214/ajr.131.2.219 PMid:97997

7. Ripollés T, Miguel-Dasit A, Errando J, Morote V, Gómez-Abril SA Richart J. Gallstone ileus: Increased diagnostic sensitivity by combining plain film and ultrasound. Abdom Imaging. 2001;26(4):401-5. https://doi.org/10.1007/s002610000190 PMid:11441553 